\title{
An Analysis of Alterations to the SCTP RTO Calculation Mechanism for WLAN Environments
}

\author{
Sheila Fallon , Paul Jacob , Yuansong Qiao , Liam Murphy ${ }^{*}$, \\ Enda Fallon , and Austin Hanley
}

\begin{abstract}
sheila.fallon@gmail.com,pjacob@ait.ie,ysqiao@ait.ie,liam.murphy@ucd.ie,efallon@ait.ie, ahanley@ait.ie

Software Research Centre, Athlone Institute of Technology, Ireland

Performance Engineering Laboratory, University College Dublin, Ireland
\end{abstract}

\begin{abstract}
As a connection oriented transport layer protocol the Stream Control Transmission Protocol (SCTP) inherits many of the features of the Transmission Control Protocol (TCP) including the mechanism by which Retransmission Timeout (RTO) is calculated. Previous investigations have established that the mechanism through which SCTP calculates RTO is inappropriate in Wireless LAN (WLAN) environments. This paper investigates the performance implications of changes to the SCTP RTO calculation mechanism. In particular alterations to the parameters $\alpha$, the smoothing factor, and $\beta$, the delay variance factor are investigated. Results indicate that performance improvements are achievable through careful selection of $\alpha$ and $\beta$ values. Throughput improvements of $63 \%$ over the default mechanism defined in RFC 4960 are described. These performance improvements however, while significant, still can not address the switchover delays which result from the distortions caused by continuously increasing RTT values in WLAN environments.
\end{abstract}

\section{Introduction}

In recent years there has been a significant increase in the availability of wireless and mobile networks capable of supporting IP communication. The diversity of these networks in terms of signal coverage and transmission capability has made it essential to employ a transparent network migration strategy which can seamlessly 
migrate from one network to another without a perceived degradation in quality from an end user. Significant research efforts are ongoing to investigate if SCTP [1], which was originally designed as a transport protocol for Signaling System 7 (SS7) data across IP networks, can support seamless network migration. As a transport layer protocol SCTP shares some of the features of TCP, as well as introducing enhancements to support network mobility. Foremost amongst these enhancements is its support for multi-homing - the ability to implement an end to end communication session transparently over multiple physical paths where the end point of each path is identified by an IP address.

As a connection oriented transport layer protocol SCTP inherits many of the features of TCP including the mechanism by which RTO is calculated. For a traditional connection oriented protocol such as TCP the selection of an appropriate RTO value is a tradeoff between: (a) setting a large value which may degrade performance by allowing excessively long periods before retransmitting a lost packet (b) setting a conservative value which mistakenly retransmits valid packets, known as spurious retransmissions. In addition to the tradeoff between (a) excessive retransmission delay and (b) spurious retransmissions, SCTP must also consider the effect of RTO selection on switchover.

Previous investigations undertaken [2] have established that the current SCTP mechanism for calculating RTO values is inappropriate in WLAN environments, since increased Round-Trip Time (RTT) significantly distorts RTO calculations. It was illustrated that SCTP behaves in a counterintuitive manner which allows more time for switchover as network conditions degraded: delays of up to 187 seconds were experienced before switchover occurred. This paper investigates if it is possible to alleviate the switchover performance deficiency experienced in WLAN environments through configuration of the parameters $\alpha$, the smoothing factor, and $\beta$, the delay variance factor.

This paper is organized as follows. Section 2 details related work in the area. Section 3 describes SCTP path management functionality. Section 4 describes the SCTP RTO calculation mechanism and illustrates the importance of the parameters $\alpha$ and $\beta$. In Section 5 some background is provided on how increased RTT in can affect SCTP RTO calculation. In Section 6 results are presented which illustrate the performance implications of changes to $\alpha$ and $\beta$. Finally conclusions and future work are discussed in Section 7.

\section{Related Work}

The mechanism by which SCTP calculates the value of a retransmission timer is inherited from TCP. The TCP mechanism itself is defined in [3] and has developed over time. In response to Internet "congestion collapse" of the mid 1980s it was suggested [4] that a back off mechanism be employed when congestion was detected. The suggested alterations were formally adopted [5] in 1989. In [4] the 
values .25 and .125 were suggested for the estimator gains and variation weight respectively. In [6] Karn's algorithm suggested that RTT measurements should not be taken for retransmitted packets. In [7] it was suggested that TCP connections utilizing large congestion windows should, if possible, take at least one RTT measurement per RTT in order to improve estimation. A mechanism which TCP should employ to begin sending after RTO expiration is outlined in [8].

With the advent of wireless networking a number of investigations have focused on accurate RTO estimation. In [9] results are presented which indicate that the optimal RTO, which maximizes TCP throughput, should take into consideration the TCP window size as well as RTT. In [10] the impact of variable transmission delays, as experienced in wireless and mobile networks, on TCP performance are investigated. In particular TCPs RTT estimation for bulk data traffic over wireless links is analysed. The results indicate that the RTT sampling rate has a significant impact on performance. In [11] optimizations for a TCP sender in the presence of delay spikes are discussed. The authors recommend timing every segment and restarting the retransmit timer to achieve a more conservative RTO estimate. In [12] the behaviour of TCP during vertical network switchover from a high capacity to low capacity network is investigated. The investigation concentrates on the TCP timeout problem caused by increased network RTT. Three schemes; fast response, slow response and ACK delay are evaluated. Simulation results presented demonstrated that these schemes can improve the performance of TCP during soft vertical handover. In [13] the performance of SCTP, TCP, and Eifel are compared during delay spikes. Results indicate that in the presence of delay spikes without packet loss, SCTP and TCP Reno have similar performance. This paper does not consider continuously increasing RTT.

A number of studies have been undertaken which investigate the performance of SCTP switchover. In [14] an analytical study of SCTP failover is undertaken which indicates that the current mechanism for calculating the duration of an SCTP switchover is unsatisfactory. Two additional parameters are introduced to the SCTP failover strategy in order to more accurately reflect the exact time at which catastrophic primary path failure occurs. In [15] performance implications of the use of heterogeneous wireless networks with differing bandwidths are presented. In [16] it is suggested that the SCTP handover strategy is reactive in nature and a more proactive approach where handover is based on path delays should be introduced in order to pre-empt and avoid path failures. In [17] it is suggested that SCTP path failure detection is inadequate for wireless networks. A new path failure detection method which utilises cycle sampling rather than the single sampling method as suggested in [1] is evaluated. Results presented indicate that the cycle sampling method can increase performance. In [18] a new scheme for primary path management for the mobile terminals during SCTP handover is evaluated. The proposed scheme utilises the absolute gap and relative ratio of the RTT of the primary and alternate paths in the switch decision. The proposed scheme implements conservative and aggressive modes of operation depending on the absolute gap of the measured RTTs in the network. The results presented indicate that a 
conservative scheme is preferred for a network where the gaps of the measured RTTs are small, whereas an aggressive scheme needs to be considered in a network when the gaps of RTTs are relatively large.

\section{SCTP Path Management}

SCTP identifies a path by the IP address of the destination. During the protocol startup stage, each SCTP endpoint selects one of its peer's IP addresses as a primary path for data transmission. When the primary path fails, a backup path will be selected as primary path. SCTP sends heartbeat packets periodically to an idle address to detect the reachability of the address. SCTP defines "idle address" as: no data chunks are sent within the current heartbeat interval. An address is considered active if the sender received the expected acknowledgement from its peer within a designated period. Otherwise, if the number of consecutive transmission timeouts exceeds the protocol parameter Path.Max.Retrans (PMR), it means the address is inactive. If this inactive address is current primary path, a handover will occur. The SCTP parameters which are used to implement the switch over management strategy are; RTO.Initial $=3$ seconds, RTO.Min $=1$ second, RTO.Max = 60 seconds and Path.Max.Retrans $=5$ attempts.

\section{RTO Calculation in SCTP}

RTO is the time that elapses after a packet has been sent until the sender considers it lost and retransmits it. In this way RTO is a prediction of the upper limit of RTT. If an SCTP sender does not receive a response for an SCTP data chunk from its receiver within the time of RTO, the sender will consider this data chunk lost. According to [1] RTO is calculated for each destination address separately based on the Smoothed Round-Trip Time (SRTT) and Round-Trip Time Variation (RTTVAR) of the path. It is initialized with RTO.Initial which is an SCTP parameter and can be configured by the user:

RTO = RTO.Initial

SRTT and RTTVAR of a path are calculated by the measurement of RTT of the path. The RTT measurement for a path is made for every round trip. When SCTP gets the first measurement of RTT: RTT.1st, SRTT and RTTVAR are initialized as:

SRTT $=$ RTT..$^{\text {st }}$

$R T T V A R=R T T \cdot 1 s t / 2$ 
And RTO is updated to

$\mathrm{RTO}=\mathrm{SRTT}+4 \times \mathrm{RTTVAR}$

For each time SCTP gets a new measurement of RTT: RTT.new, SRTT and RTTVAR will be updated as follows:

RTTVAR.new $=(1-\beta) \times$ RTTVAR.old $+\beta \times($ SRTT.old - RTT.new $)$

SRTT.new $=(1-\alpha) \times$ SRTT.old $+\alpha \times$ RTT.new

Where $\beta$ and $\alpha$ are constants and their recommended values are $1 / 4$ and $1 / 8$ respectively. Then the new RTO is:

RTO $=$ SRTT.new $+4 \times$ RTTVAR.new

If the new RTO is less than RTO.Min, it will be set to RTO.Min. If the new RTO is greater than RTO.Max, it will be set to RTO.Max. Every time a transmission timeout occurs for an address, the RTO for this address will be doubled:

$\mathrm{RTO}=\mathrm{RTO} \times 2$

And if the new RTO is greater than RTO.Max, RTO.Max will be used for the new RTO. If the sender gets a response from the receiver and a new RTT is measured, SCTP will use this new RTT to calculate RTTVAR, SRTT and finally RTO by the equations (3.5) to (3.7).

\section{An Illustration of the Effect of Increased RTT on SCTP RTO Calculation}

In this section a brief illustration of the effect of increased RTT experience in a WLAN environment on RTO estimation in SCTP is presented. A more complete description of the performance implications of increased RTT on SCTP RTO can be found in [2]. In the following example a mobile node is communicating with a back end server through a Linksys WRT54GL 802.11g access point. The test starts with the mobile node adjacent to the access point. The mobile node then moves at slow walking pace away from the access point. As the mobile node moves from the coverage area of the AP signal strength degrades and results in intermittent network connectivity. After 100 seconds the RTT increases significantly. Figure 1 details the increased RTT and illustrates its affect on RTO calculation through the SRTT (3.6) and RTTVAR (3.7) parameters. 


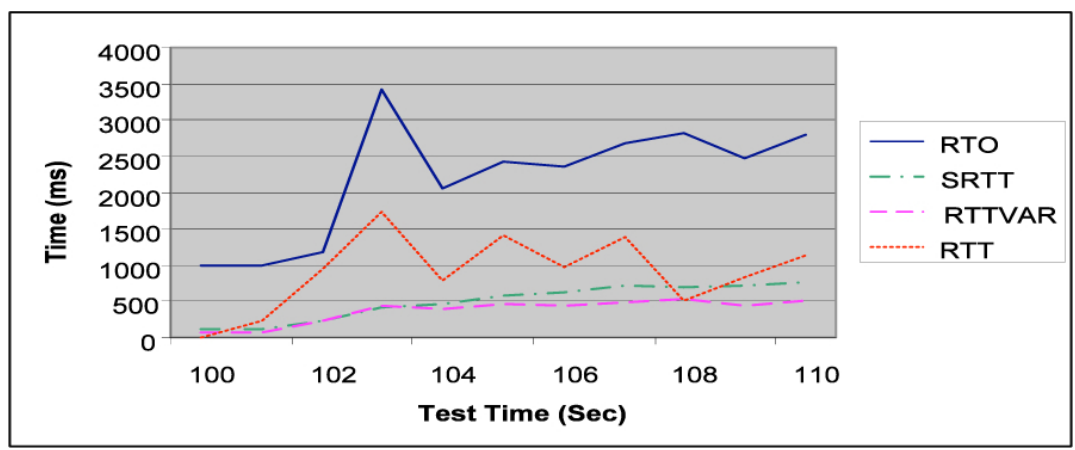

Fig. 1 RTT and RTO Values for Time 100-110 Secs

For the period shown the calculation of RTO is based on the recorded RTT value by applying (3.5) (3.6) and (3.7). As a result of the continuously high RTT value the baseline RTO value also increases significantly. As the packet retransmission failures occur (3.8) is applied which doubles an already high baseline RTO value.

After 100 seconds the RTO was $1000 \mathrm{~ms}$, SRTT was $107 \mathrm{~ms}$, RTTVAR was $79 \mathrm{~ms}$ and the recorded RTT was $7 \mathrm{~ms}$. Within a period of 10 seconds the RTO has increased from $1000 \mathrm{~ms}$ to $2802 \mathrm{~ms}$. The average RTO between 100 and $250 \mathrm{sec}-$ onds was 3.148 seconds. If a retransmission timeout occurred during this time period (3.8) would be applied. This would double the RTO to $5602 \mathrm{~ms}$. Using the default PMR value of 5 it would take $2.8+5.6+11.2+22.4+44.8+60=146.8$ seconds for switchover to occur. Even using more aggressive PMR values of 0,1 and 2 excessive delays of 2.8, 8.4 and 19.6 seconds respectively are experienced.

\section{Results}

In order to investigate the performance implications of changes to $\alpha$, the smoothing factor, and $\beta$, the delay variance factor on SCTP RTO estimation and performance a number of studies were undertaken as follows:

- An experimental study which illustrates that mobility can result in continually increasing RTT in a WLAN environment

- An analytical study which considers the optimal configuration of $\alpha$ and $\beta$ for a traditional TCP oriented trade off between (a) excessive retransmission delay and (b) spurious retransmissions in the presence of continuously increasing RTT.

- A simulated study which considers the optimal configuration of $\alpha$ and $\beta$ when the effects of SCTP path switchover are considered in the presence of continuously increasing RTT. 
The aim of these investigations was to determine if the performance deficiencies relating to path switchover highlighted in [2] could be addressed through careful configuration of the $\alpha$, and $\beta$ parameters employed in the estimation of RTO.

\subsection{Experimental Illustration of Increased RTT in WLAN}

From equations (3.2) to (3.6) in can be seen that RTO is significantly dependent on RTT. In order to estimate RTT and loss rates in wireless environments 25 tests were undertaken which utilized Ixia's IXChariot [19] network analysis software which transmitted RTP data using H263QCIF service quality at a constant bit rate of $3.75 \mathrm{Mbps}$. The tests were initiated adjacent to the access point. The mobile client then moved at slow walking pace, approximately $1 \mathrm{~m} / \mathrm{sec}$, away from the access point. Figure 2 illustrates the average RTT and loss rates for 25 tests.

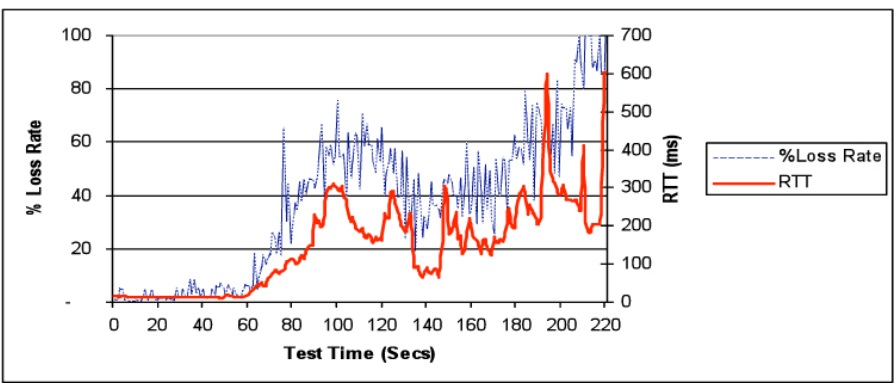

Fig. 2 Average RTT and Loss Rates in WLAN Environment

Figure 2 illustrates that as the mobile node moved from the coverage of the access point the RTT and loss rate increased significantly, particularly after $60 \mathrm{sec}-$ onds.

\subsection{A TCP Oriented Analysis of the Optimal Configuration of $\alpha$ and $\beta$ With Increasing RTT in WLAN}

In order to analyse the optimal configuration of $\alpha$ and $\beta$ in TCP oriented manner and to address the tradeoff between (a) excessive retransmission delay and (b) spurious retransmissions for a WLAN environment an analytical study was undertaken. This analytical study used the average RTT estimated by the experimental study, illustrated in Figure 2, as input. The analytical study employed the following configurations of $\alpha$ and $\beta$ pairs: 


$$
\alpha=i, \beta=j\{i, j \in 0, .1, .2, \ldots \ldots, 1.0\}
$$

As an example, Figure 3 illustrates how different configurations of $\alpha$ and $\beta$ affect RTO estimation. It illustrates how the estimated RTO deviates from RTT. The X-axis represents the baseline RTT values illustrated in Figure 3. Positive values indicate that the RTO estimation is greater than the actual RTT, in such a scenario excessive delays occur before lost packet retransmission. Negative values indicate that the RTO estimation is less than actual RTT, in such a scenario spurious retransmissions result.

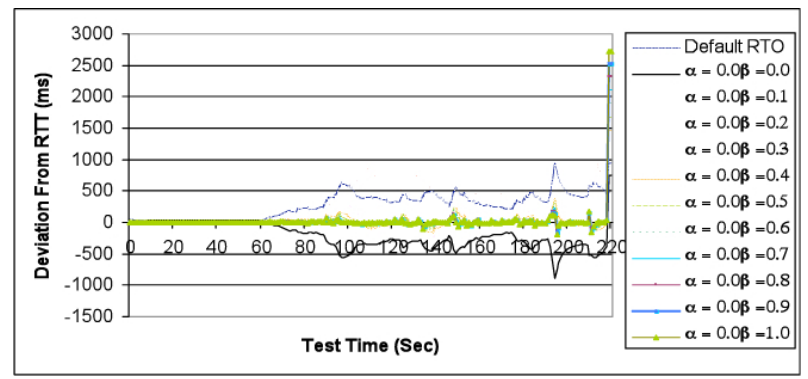

Fig. 3 RTO Deviations From RTT for $\alpha=0$ and $\beta$ ranging from $0, .1, \ldots 1$

Figure 4 illustrates the accumulated potential delay before lost packet retransmission, for each $\alpha$ and $\beta$ pair i.e. the area above the $\mathrm{x}$-axis, calculated as follows:

$$
\sum_{i=0}^{i=220}\left(R T O_{i}-R T T_{i}\right)
$$

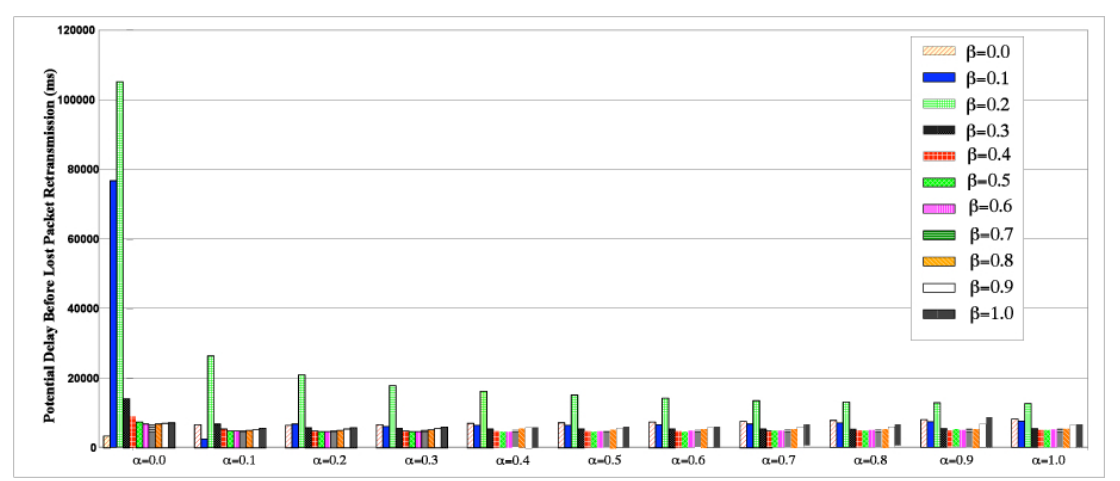

Fig. 4 Potential Delay Before Lost Packet Retransmission for $\alpha$ and $\beta$ pairs

Figure 4 illustrates that $\alpha=.1 \beta=.1$ and $\alpha=0 \beta=0$ were the most effective test pairs with potential delays of 2.59 and 3.4 seconds respectively. The test pairs with the greatest delay were $\alpha=0 \beta=.1$ and $\alpha=0 \beta=.2$ with delays of 76.74 and 105.11 seconds respectively. 
Figure 5 is concerned with the estimation of the total number of spurious retransmissions. It illustrates the potential number of seconds in which spurious retransmission would occur and is calculated as follows:

$$
\sum_{i=0}^{i=220} \text { if }\left(R T O_{i}<R T T_{i}\right) 1, \text { else } 0
$$

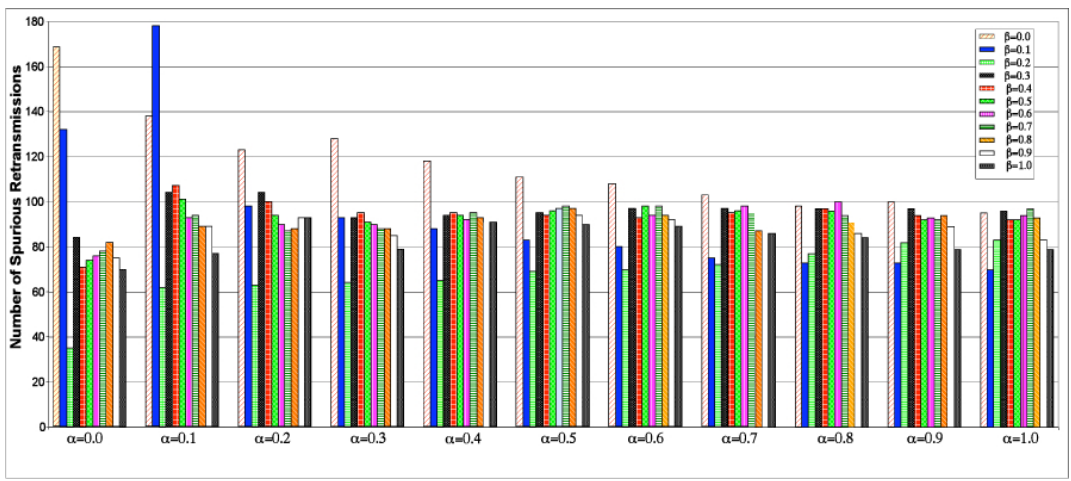

Fig. 5 Number of Seconds in which Spurious Retransmissions Occur for $\alpha$ and $\beta$ pairs

Figure 5 illustrates that the test pairs with least number of spurious retransmissions were $\alpha=0.0 \beta=0.2, \alpha=0.1 \beta=0.2$ and $\alpha=0.2 \beta=0.2$ with 35,62 and 63 seconds of spurious retransmissions respectively. The test pairs with the most seconds of spurious retransmissions were $\alpha=.1 \quad \beta=.1, \alpha=0 \beta=0$ and $\alpha=$ $.1 \beta=.1$ with 178,169 and 138 seconds of spurious retransmissions respectively.

In order to determine the optimal selection of $\alpha$ and $\beta$ each of the test pairs were graded from 1 to 121 in terms of efficiency relating to lost packet retransmission delay and spurious retransmission. As an example the test pair $\alpha=0.0 \beta=0.2$ had the least number of spurious retransmissions $(\mathrm{x}=0)$ yet the highest potential delay before lost packet retransmission $(y=120)$ thus resulting in the point $(0,120)$. Figure 6 illustrates the performance distribution of test pairs.

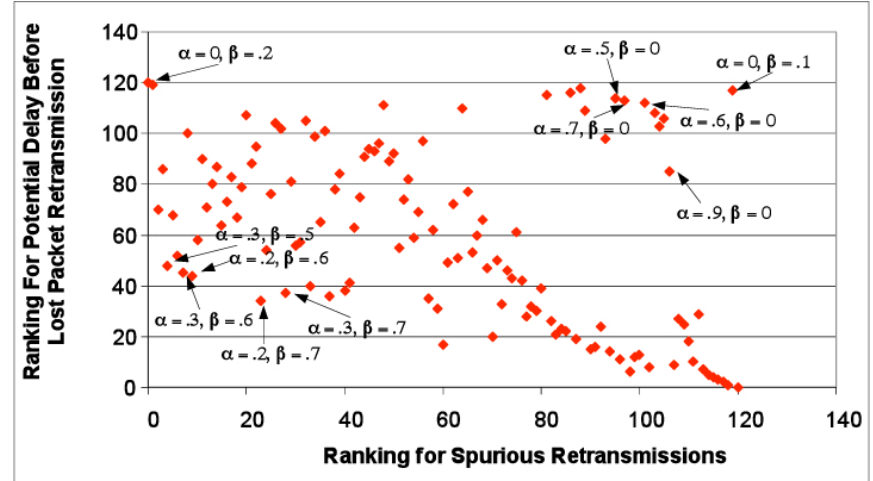

Fig. 6 Performance Distribution for $\alpha$ and $\beta$ pairs 
For each test pair the Euclidean distance from the origin was calculated. Table 1 illustrates that the test pairs likely to provide the optimal tradeoff between excessively long periods before retransmitting a lost packet and spurious retransmission of valid packets were $\alpha=0.2 \beta=0.7, \alpha=0.2 \beta=0.6$ and $\alpha=0.3 \beta=0.6$.

Table 1 Performance Ranking for $\alpha$ and $\beta$ pairs

\begin{tabular}{|r|l|r|r|l|r|}
\hline Ranking & Pair Values & $\begin{array}{r}\text { Euclidean } \\
\text { Distance }\end{array}$ & Ranking & Pair Values & $\begin{array}{r}\text { Euclidean } \\
\text { Distance }\end{array}$ \\
\hline $\mathbf{1}$ & $\alpha=0.2 \beta=0.7$ & 41.05 & $\mathbf{1 1 7}$ & $\alpha=0.5 \beta=0.0$ & 148.92 \\
\hline $\mathbf{2}$ & $\alpha=0.2 \beta=0.6$ & 44.91 & $\mathbf{1 1 8}$ & $\alpha=0.9 \beta=0.0$ & 149.20 \\
\hline $\mathbf{3}$ & $\alpha=0.3 \beta=0.6$ & 45.54 & $\mathbf{1 1 9}$ & $\alpha=0.7 \beta=0.0$ & 149.24 \\
\hline $\mathbf{4}$ & $\alpha=0.3 \beta=0.7$ & 46.40 & $\mathbf{1 2 0}$ & $\alpha=0.6 \beta=0.0$ & 150.81 \\
\hline $\mathbf{5}$ & $\alpha=0.3 \beta=0.5$ & 48.17 & $\mathbf{1 2 1}$ & $\alpha=0.0 \beta=0.1$ & 166.88 \\
\hline
\end{tabular}

\subsection{Analysis of the Optimal Configuration of $\alpha$ and $\beta$ with SCTP Path Switchover and Increasing RTT in WLAN}

The analytical study described in Section 6.2 suggested optimal configurations for $\alpha$ and $\beta$ parameters in order to provide a tradeoff between (a) excessive retransmission delay and (b) spurious retransmissions. The analytical study however, did not consider the affect of path switchover on performance. In this section the effect of path switchover is considered.

The same $\alpha$ and $\beta$ pair configurations as in the analytical study were evaluated using the University of Delewares [20] SCTP module for NS2 [21]. The simulation topology is detailed in Figure 7.

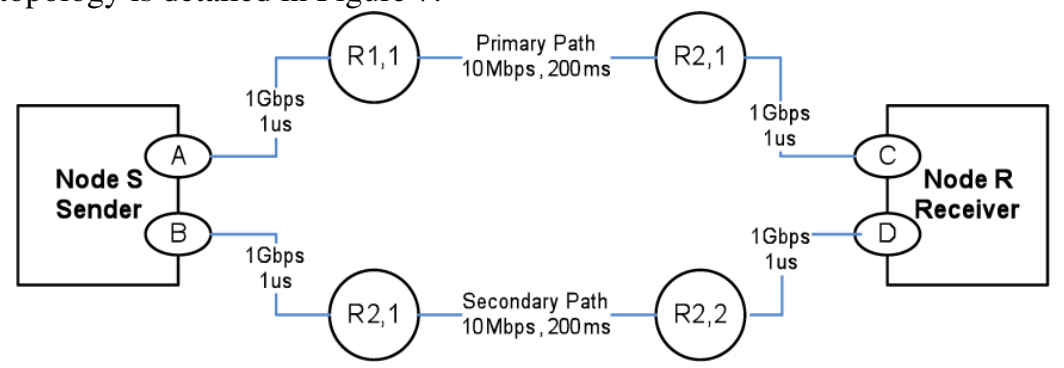

Fig. 7 Simulation Configuration

Node S and Node R are SCTP sender and receiver respectively. Both SCTP endpoints have two addresses. R1,1, R1,2, R2,1 and R2,2 are routers. The implementation is configured with no overlap between the two paths. Node $\mathrm{S}$ begins to send FTP data to Node R after 0.5 seconds. 
As with the analytical study the simulations used the average RTT values, illustrated in Figure 2 as input. Since $\alpha$ and $\beta$ are not configurable SCTP parameters their alteration required updates to and recompilation of [20] for each test. Each test pair was ranked by its performance in relation to the total amount of data transmitted. Table 2 illustrates the variation in performance for a representative sample of the configurations, between the analytical study and the simulated investigation.

Table 2 Performance Comparison for $\alpha$ and $\beta$ pairs for Analytical and Simulated Study

\begin{tabular}{|c|c|c|c|c|c|}
\hline \multirow{2}{*}{$\begin{array}{c}\text { Pair } \\
\text { Values }\end{array}$} & \multicolumn{2}{|c|}{ Ranking } & \multirow{2}{*}{$\begin{array}{c}\text { Pair } \\
\text { Values }\end{array}$} & \multicolumn{2}{|c|}{ Ranking } \\
\hline & Simulated & Analytical & & Simulated & Analytical \\
\hline$\alpha=0.7 \beta=0.7$ & 1 & 104 & $\alpha=0.8 \beta=0.5$ & 117 & 49 \\
\hline$\alpha=0.9 \beta=0.7$ & 2 & 121 & $\alpha=0.0 \beta=0.7$ & 118 & 96 \\
\hline$\alpha=1.0 \beta=0.7$ & 3 & 105 & $\alpha=0.9 \beta=0.9$ & 119 & 65 \\
\hline$\alpha=0.1 \beta=0.8$ & 4 & 100 & $\alpha=0.5 \beta=0.7$ & 120 & 35 \\
\hline$\alpha=0.2 \beta=0.8$ & 5 & 89 & $\alpha=0.0 \beta=0.3$ & 121 & 53 \\
\hline
\end{tabular}

The results illustrated in Table 2 indicate that there is a significant variation in performance when the effect of path switchover is considered. For SCTP the selection of an appropriate RTO value is not only a tradeoff between (a) excessive retransmission delay and (b) spurious retransmissions. As a multi-homed protocol SCTP is significantly affected by the RTO value as it is used as a mechanism to determine when switchover is initiated.

Figure 8 graphs the performance rankings of the $\alpha$ and $\beta$ pairs from the most effective $\alpha=0.7 \beta=0.7$ to the least effective $\alpha=0.0 \beta=0.3$. The results indicate an inverse relationship between data throughput and switchover time; as switchover times increase data throughput degrades.

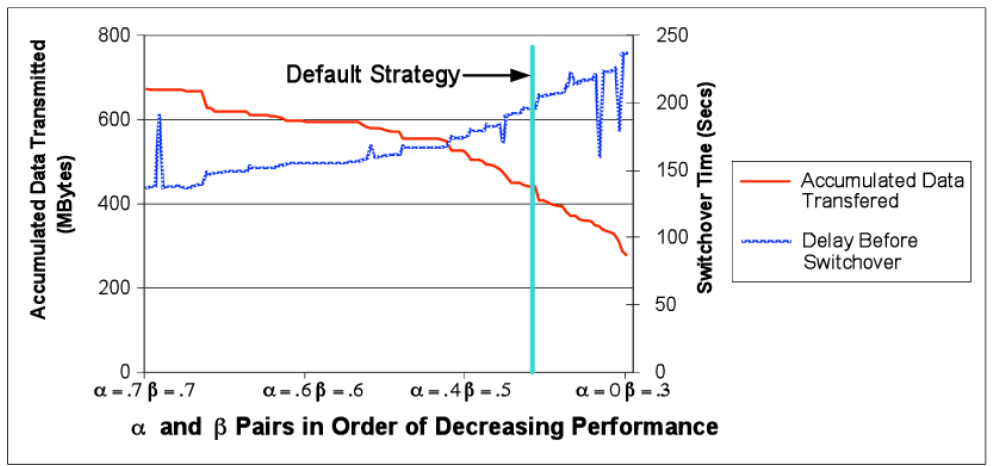

Fig. 8 Performance Comparison of Analytical and Simulated Results for $\alpha$ and $\beta$ pairs 
The default RTO calculation, which utilises the values $\alpha=.125, \beta=.25$, transmitted 412 Mbytes of data. The 3 pair configurations which were selected by the analytical study as the optimal tradeoff between (a) excessive retransmission delay and (b) spurious retransmissions $\alpha=.2, \beta=.7, \alpha=.2, \beta=.6$ and $\alpha=.3, \beta=.6$ transmitted 527, 527 and 359 MBytes respectively.

When the effects of switchover were considered by the simulated study the 3 most effective strategies were $\alpha=.7, \beta=.7, \alpha=.9, \beta=.7$ and $\alpha=1, \beta=.7$. These configurations transmitted 673, 672 and 671 Mbytes respectively which was significantly more than the default configuration. Figure 9 compares the performance of the default strategy against the 3 most effective configurations from the analytical and simulated studies.

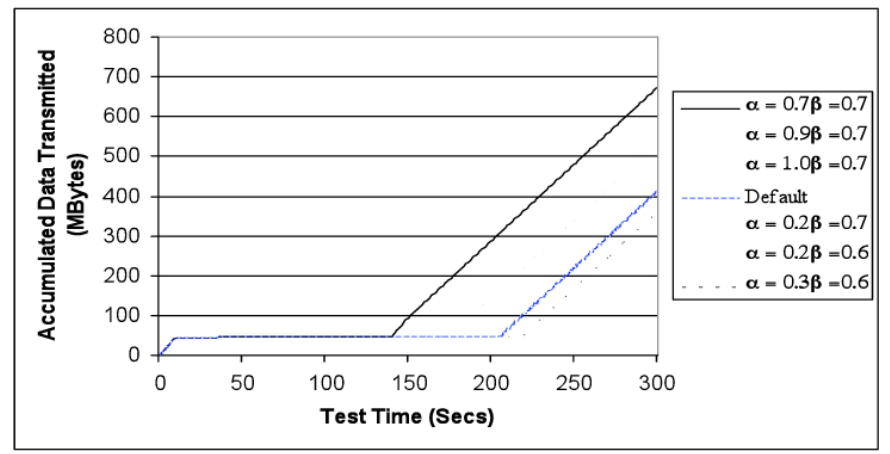

Fig. 9 Accumulated Data Transmitted for Selected $\alpha$ and $\beta$ pairs

Figure 9 illustrates that alteration of $\alpha$ and $\beta$ pairs from the default have the potential to improve SCTP throughput by up to $63 \%$. This performance improvement is achieved through reduced switchover time due the altered $\alpha$ and $\beta$ configurations. However there remains a significant "flat line" between approximately 30 seconds and 140 seconds as a result of the switchover delay due to excessively large RTO described in Section 5.

\section{Conclusion and Future Work}

This paper investigated how changes to the SCTP RTO calculation mechanism, in particular alterations to the parameters $\alpha$, the smoothing factor, and $\beta$, the delay variance factor affected SCTP performance in a WLAN environment.

Experimental investigations illustrated how mobility resulted in continually increasing RTT in a WLAN environment. Using these results as input an analytical study was undertaken that considered the optimal configuration of $\alpha$ and $\beta$ in the presence of continuously increasing RTT. The analytical study considered the traditional TCP oriented trade off between (a) excessive retransmission delay and (b) 
spurious retransmissions. The results indicated the optimal configurations for $\alpha$ and $\beta$ in the presence of continuously increasing RTT. These optimal values of $\alpha=.2, \beta=.7, \alpha=.2, \beta=.6$ and $\alpha=.3, \beta=.6$ differ significantly from the default values of $\alpha=.125, \beta=.25$.

For SCTP however, the selection of an appropriate RTO value is not only a tradeoff between (a) excessive retransmission delay and (b) spurious retransmissions, SCTP must also consider the effect of RTO selection on switchover. Therefore a simulated study was then undertaken to consider the optimal configuration of $\alpha$ and $\beta$ when the effects of SCTP path switchover were considered in the presence of continuously increasing RTT. The results indicated a significant variation in performance between the analytical and simulated studies. Two of the three $\alpha$ and $\beta$ configurations which were selected as optimal by the analytical study were found to be more effective than the default strategy. However, the three most effective configurations suggested by the simulated study were $\alpha=0.7 \beta=0.7, \alpha=0.9 \beta=0.7$ and $\alpha=1.0 \beta=0.7$. These configurations transmitted significantly more than the configurations suggested by the analytical study and up to $63 \%$ more data than the default configuration as defined in RFC 4960.

While the results indicate that performance improvements are possible as a result of reduced switchover time through careful $\alpha$ and $\beta$ configuration there remains a significant switchover delay due to excessively large RTO.

Future work will investigate optimized mechanisms for SCTP path management in WLAN environments. One mechanism may involve an SCTP switch management algorithm which will recognize continuously increasing RTT as an indicator of imminent path failure in a WLAN environment. Another approach may investigate a cross layer switch management strategy which utilizes received signal strength as a parameter in the path selection decision.

\section{References}

1. R. Stewart et al. Stream Control Transmission Protocol, RFC 4960, Sep. 2007.

2. Fallon, S et al. A "SCTP Switchover Performance Issues in WLAN Environments", IEEE Consumer Communications \& Networking Conference (CCNC) 2008

3. Allman, M., Paxson V. "Computing TCP's Retransmission Timer" RFC 2988 November 2000

4. Jacobson, V., "Congestion Avoidance and Control", Computer Communication Review, vol. 18, no. 4, pp. 314-329, Aug.

5. Braden, R., "Requirements for Internet Hosts - Communication Layers", RFC 1122, October 1989.

6. Karn, P. and C. Partridge, "Improving Round-Trip Time Estimates in Reliable Transport Protocols", SIGCOMM 87.

7. Jacobson V.Braden R. Borman. D "TCP Extensions for High Performance” May 1992

8. Allman, M., Paxson V. and W. Stevens, "TCP Congestion Control", RFC 2581, April 1999.

9. Kesselman, A, Mansourz, Y, “Optimizing TCP Retransmission Timeout", 4th International Conference on Networking, Reunion Island, France 2005 
10. Scharf, M, Necker, M, Gloss, B, “The Sensitivity of TCP to Sudden Delay Variations in Mobile Networks" Lectute notes in Computer Science 2004

11. Gurtov, A, "Effect of Delays on TCP Performance" IFIP Personal Wireless Communications 2001, Lappeenranta, Finland

12. Huang, H, Cai, J, "Improving TCP performance during soft vertical handoff", 19th International Conference on Advanced Information Networking and Applications, 2005.

13. Fu, S, Atiquzzaman, M, Ivancic, W, "Effect of delay spike on SCTP, TCP Reno, and Eifel in a wireless mobile environment" Conference on Computer Communications and Networks, 2002.

14. Budzisz L et al. "An Analytical Estimation of the Failover Time in SCTP Multihoming Scenarios" Wireless Communications and Networking Conference (WCNC) 2007

15. Qiao Y et al. "SCTP Performance Issue on Path Delay Differential", Wired/Wireless Internet Communications (WWIC) 2007

16. Kelly, A, et al. "Delay-Centric Handover in SCTP over WLAN", Transactions on Automatic Control and Computer Science, 49, 63 (2004), 1--6.

17. Min-Chin, C, Jen-Yi, P, Ting-Wei, H, "A Smart Path Failure Detection Method for SCTP in Wireless Network" International Conference on Wireless Communications, Networking and Mobile Computing, 2007. WiCom 2007.

18. Kim, D, Koh, S, Kim, Y, “A Scheme of Primary Path Switching for Mobile Terminals Using SCTP Handover" 2007 annual Conference on International Conference on Computer Engineering and Applications

19. IXChariot Console version 6.50 www.ixia.com

20. A. Caro, et al : ns-2 SCTP module, Version 3.5, www.armandocaro.net/software/ns2sctp/.

21. G. Combs, et al : Wireshark network protocol Analyzer, Version 0.99.5, www.wireshark.org 